\title{
La perception des inégalités en France
}

Essai d'explication

\section{Olivier Galland, Yannick Lemel et Alexandra Frénod}

\section{(2) OpenEdition}

\section{Journals}

Édition électronique

URL : http://journals.openedition.org/ress/2343

DOI : $10.4000 /$ ress.2343

ISSN : 1663-4446

Éditeur

Librairie Droz

\section{Édition imprimée}

Date de publication : 1 juin 2013

Pagination : 179-211

ISBN : 978-2-600-01749-7

ISSN : 0048-8046

\section{Référence électronique}

Olivier Galland, Yannick Lemel et Alexandra Frénod, «La perception des inégalités en France », Revue européenne des sciences sociales [En ligne], 51-1 | 2013, mis en ligne le 01 janvier 2017, consulté le 30 avril 2019. URL : http://journals.openedition.org/ress/2343 ; DOI : 10.4000/ress.2343 


\title{
LA PERCEPTION DES INÉGALITÉS EN FRANCE
}

ESSAI D'EXPLICATION

\author{
OLIVIER GALLAND \& YANNICK LEMEL \\ Avec la collaboration d'ALEXANDRA FRÉNOD \\ GEMASS (Paris-IV Sorbonne) \& GEMASS (Paris-IV Sorbonne) / CREST \\ ogalland@msh-paris.fr/lemel@ensae.fr/afrenod@msh-paris.fr
}

\begin{abstract}
Résumé. Cet article vise à tester trois théories explicatives de la perception des inégalités sociales en se fondant sur une enquête par questionnaire complétée par une série d'entretiens qualitatifs. Nous examinerons la théorie de «l'intérêt bien compris» lié à la position sociale, celle de l'effet éventuel de mécanismes de frustration relative, et celle de l'effet de l'adhésion à des valeurs en matière de justice sociale. Les résultats montrent des effets contrastés des différentes variables explicatives selon le niveau de perception des inégalités. La position sociale comme les mécanismes de frustration relative expliquent bien l'impact personnellement ressenti, alors qu'ils n'exercent pratiquement aucun effet sur la perception globale des inégalités sociales. La perception de l'inégalité dans la société est en revanche associée, quelle que soit la position sociale, à des principes en matière de justice. Ce n'est pas la position des individus dans la stratification sociale qui informe leur perception des inégalités, mais des principes qui en sont largement indépendants.
\end{abstract}

Mots-clés: inégalités, justice sociale, statut social, frustration relative, discrimination, stratification sociale.

\begin{abstract}
This article aims to test three explanatory theories of the perception of social inequalities, on the basis of a quantitative survey, completed by qualitative interviews. We will examine the theory of "in the best interests" linked to social position, the theory of the possible effect of relative deprivation mechanisms and the theory of values in terms of social justice. The results show contrasting effects of the different explanatory variables according to the perception level of inequality. Social status as well as relative deprivation mechanisms explain the personally felt impact, whereas they have practically no effect on the wider perception of social inequalities. On the other hand the perception of inequalities in society is associated, whatever the subject's social status, with principles of justice. It is not the position of individuals in the system of social stratification that informs their perception of inequalities, but rather principles that are largely independent of their social status.
\end{abstract}

Keywords: inequality, social justice, social status, relative deprivation, discrimination, social stratification. 
Les Français ont le sentiment d'appartenir à une société très inégalitaire. Plusieurs recherches (Cherkaoui, I988 ; Piketty, 1999; Godechot, Gurgand, 2000 ; Hadler, 2005; Forsé, Galland, 20II), confirment ce résultat que des enquêtes précédentes avaient déjà mis en lumière, notamment une précédente enquête du GEMAS (1976) qui portait également sur la question de la perception des inégalités sociales, et l'enquête ISSP 1999 consacrée à la représentation des inégalités (Forsé, Parodi, 20Io). Cet article vise à mieux comprendre les ressorts de ces perceptions en s'appuyant sur un double matériau, quantitatif et qualitatif. Il cherche à évaluer diverses théories explicatives de ces perceptions, notamment en examinant les relations entre les composantes de la position sociale occupée par les individus et leurs perceptions des différentes facettes des inégalités, ainsi que l'effet exercé par l’adhésion à des principes de justice sociale.

\section{L'ÉTAT DES LIEUX Quelles sont les opinions des Français au sujet des inégalités sociales? \\ I.I. GLOBALEMENT, DES INÉGALITÉS DANS LA SOCIÉTÉ JUGÉES FORTES ET INTOLÉRABLES}

Une originalité de l'enquête menée sur la perception des inégalités et des sentiments de justice (dite PISJ) que cet article prolonge, est de ne pas se limiter aux seules inégalités économiques - revenu ou patrimoine -, comme on le fait usuellement, mais d'en distinguer plusieurs types. Les personnes interrogées dans l'enquête devaient ainsi donner leur opinion au sujet de douze types d'inégalités : revenu, patrimoine, emploi, éducation, accès aux soins médicaux, risques technologiques, origine ethnique, sexe, âge, insécurité, logement, pénibilité du travail. ${ }^{.}$Ces inégalités ne sont évidemment pas toutes de même nature, nous y reviendrons.

I L'enquête précédente du GEMAS comprenait également des questions sur différents types d'inégalités. 
Une autre spécificité de l'enquête est de distinguer différentes composantes de la perception des inégalités sociales par les individus. On distingue ainsi, pour chaque inégalité considérée, trois types d’appréciation: l'opinion sur l'importance objective d'un type d'inégalités dans la société tout entière que nous qualifierons dans la suite de l'article par le terme «force », l'opinion sur le caractère plus ou moins acceptable ou inacceptable d'un type donné d'inégalités dans la société que nous subsumons sous la catégorie «illégitimité», enfin, le sentiment d'être personnellement plus ou moins touché par un type donné d'inégalités que nous résumerons par l'expression «impact personnel».

Lorsqu'on examine les réponses à ces batteries de questions, un premier résultat apparaît: en première approximation, les appréciations des Français sont relativement similaires d'un type d'inégalités à l'autre. ${ }^{2}$ Les analyses en composantes principales de chacune des trois batteries - force, illégitimité et impact personnel - de questions relatives aux douze types d'inégalités, mettent en évidence, chacune, un premier facteur très largement dominant. ${ }^{3}$ Le second facteur est à la limite de la significativité. Il permet néanmoins de distinguer, on le verra plus loin, deux types d’inégalités différents.

Ce «facteur taille» qui définit donc les premières composantes des ACP justifie de construire des indicateurs globaux, respectivement de la «force», de «l'illégitimité » et de «l'impact personnel» sommant les réponses pour l'ensemble des types d'inégalités considérés. ${ }^{4}$ La figure i montre que les deux distributions des scores de la force et de l'illégitimité perçues sont largement décalées vers les

2 Dans cet article, on va être conduit à comparer les réponses données par des individus ou des groupes d'individus à des questions leur demandant de noter des situations sur des échelles (de I à I0, ou de I à 4). Faire de telles comparaisons suppose évidemment que les évaluateurs quels qu'ils soient utilisent à peu près les mêmes règles de notation, quel que soit l'objet qu'ils évaluent, hypothèse qu'on acceptera dans cet article.

3 Avec des valeurs propres comprises entre 4 et 7 selon le type d'appréciations considéré (force, illégitimité ou impact personnel).

4 L'alpha de Cronbach communément utilisé pour apprécier la fiabilité d'une échelle, est de 0,86 pour l'échelle de force des inégalités, de 0,93 pour celle d'illégitimité et de 0,84 pour l'échelle d'impact personnel. Les deux premières échelles qui somment les réponses à une batterie de 12 questions offrant dix modalités de réponse, ont donc une valeur minimum de 12 et une valeur maximum de 120 . 
notes élevées de l'échelle (variant de I à Io), la moyenne étant de 6,9 pour la force des inégalités et de 7,4 pour leur illégitimité ressentie. Les inégalités sont jugées fortes et, plus encore, inacceptables par une large partie des personnes interrogées ${ }^{5}$. La distribution de l'échelle de force est proche d'une distribution normale: les valeurs se répartissent assez également autour de la moyenne. La distribution de l'échelle d'illégitimité est plus asymétrique, les valeurs étant plus décalées vers les notes basses pour une petite partie de l'effectif et plus concentrées vers les notes élevées pour une grande partie des individus. La dispersion des opinions est donc plus marquée pour la perception de l’illégitimité des inégalités que pour la perception de leur présence dans la société. Le consensus est plus grand sur la situation des inégalités dans la société que sur leur caractère plus ou moins légitime, ce qui n’est pas forcément étonnant car la seconde opinion est plus dépendante d'orientations de valeurs que la première.

Figure I. Distribution des échelles de «force» et d'«illégitimité» des inégalités

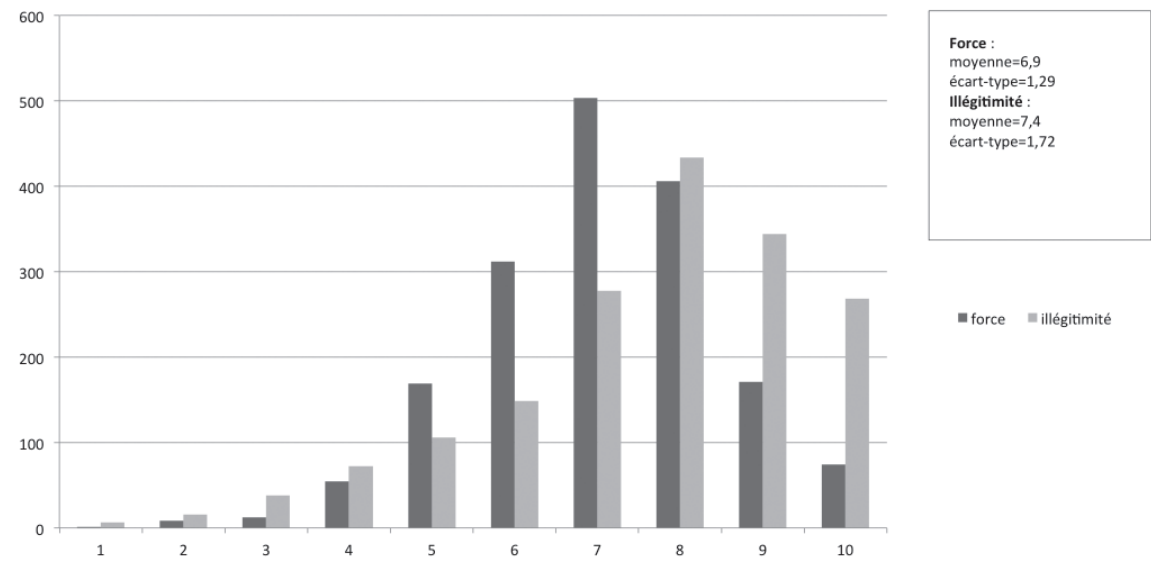

5 On retrouve bien dans les entretiens qualitatifs la même tonalité générale critique sur l'état des inégalités en France. 


\section{I.2. UN OPTIMISME PLUS GRAND SUR LA SITUATION PERSONNELLE}

Le contraste de ces réponses sur la situation globale des inégalités en France avec celles données par les personnes sur leur situation personnelle est frappant. La réponse est de 2,2 en moyenne, tous types d'inégalités confondus sur une échelle de I à 4, 4 pour la réponse «très défavorisé »" Les Français jugent en moyenne leur situation personnelle plutôt bonne, bien meilleure qu'ils ne jugent la situation de la société dans son ensemble. Cependant, la variabilité de leurs opinions sur leur situation personnelle est sans doute plus grande que celles qui concernent la société, comme on le verra plus loin et on constate d'ailleurs que le premier facteur de l'analyse en composantes principales de la batterie de questions correspondantes est moins important (36\% de la variance seulement, facteur suivant $8 \%$ ) même si une échelle globale de fort bonne qualité peut être construite en sommant tous les items.

Ces premiers résultats sont confirmés par les entretiens qualitatifs. De manière générale, la situation personnelle est en effet jugée meilleure que la situation générale même si les raisons d'en juger ainsi sont assez variées. Certaines personnes interrogées font simplement état de la satisfaction des besoins essentiels ou de la satisfaction retirée de la vie professionnelle comme des éléments suffisants pour se sentir plutôt favorisés. Nombreux sont ceux qui considèrent également que les choix qu'ils ont faits dans l'orientation de leur vie étaient associés dès le départ à un certain niveau de revenu ou plus large-

6 La question sur «l'impact personnel» des inégalités offrait volontairement des choix de positionnements moins nombreux (très favorisé, plutôt favorisé, assez défavorisé, très défavorisé) que ce n'était le cas pour les appréciations sur la société globale, l'idée étant d'offrir des choix plus explicites que ne peut l'être une simple notation. Par ailleurs, la possibilité de se déclarer «non concerné» était donnée aux personnes interrogées sur le degré auquel elles étaient personnellement touchées par les différents types d'inégalités. Exclure des analyses toutes les personnes ayant déclaré au moins une fois n'être pas concernées par une inégalité aurait nettement réduit l'échantillon. Il a donc fallu imputer ces réponses «non concerné». Diverses options étaient possibles. Après examen des résultats d'une analyse des correspondances multiples montrant que les modalités «non concerné» se positionnent avec les modalités intermédiaires, nous avons considéré que donner une telle réponse pouvait en fait s'assimiler à la situation moyenne et avons donc recodé les réponses en conséquence. Les réponses (avant comme après recodage) sont fortement corrélées et on peut créer un score global en additionnant toutes les réponses. L'alpha de Cronbach de l'échelle ainsi constituée est de 0,84. 
ment à certaines conditions de vie. Ce sentiment d'avoir fait des choix en toute connaissance de cause, atténue donc très fortement et très consciemment leur sentiment d'iniquité. Selon eux, en effet, on ne peut revendiquer des avantages connus pour être inatteignables dans le type de vie qu'on a choisi : «on est maître de son destin, il ne faut pas se positionner en victime » argumententils. Beaucoup de personnes interviewées, tout en dénonçant la présence des inégalités dans la société, reconnaissent qu’elles sont elles-mêmes épargnées et que, somme toute, la société française est plutôt juste, considérant qu'il vaut mieux y vivre que dans d'autres pays parce que le degré de protection sociale y est élevé, même s'il a tendance à être aujourd'hui fragilisé.

\section{I.3. DEUX TYPES D'INÉGALITÉS}

Accès aux biens sociaux et discriminations

Le premier facteur de l'analyse en composantes principales (Tableau I) montre que les jugements portés sur les différents types d’inégalités (revenu, patrimoine, face à l'emploi, aux études, etc..) sont assez similaires : globalement, on l'a dit, les inégalités, quelles qu'elles soient, sont jugées fortes et condamnables. Ce constat global établi, des nuances apparaissent néanmoins dans les jugements, comme le montre le deuxième facteur de l'analyse en composantes principales (Tableau I). Celui-ci oppose revenu et patrimoine (et à un moindre degré le chômage) aux inégalités liées au genre, à l'âge, à l'origine ethnique, aux risques technologiques, à l'insécurité et à un moindre degré aux soins médicaux. Cette opposition est assez cohérente d'un point de vue analytique. On voit qu'elle correspond à une différenciation entre des inégalités d’accès à des biens sociaux (revenu, patrimoine, emploi, etc.) et des discriminations (inégalités entre des catégories d'individus, hommes et femmes, jeunes et personnes plus âgées, nationaux et immigrés, etc.). On notera que risques technologiques et insécurité sont dans ce deuxième ensemble, probablement parce qu'ils se rapprochent plus d'un effet discriminatoire (un lieu de résidence, par exemple, peut être associé à des risques technologiques spécifiques que ne connaissent pas des personnes habitant dans d'autres endroits mais qui frappent uniformément toutes les personnes 
vivant au même endroit. Des commentaires de ce type apparaissent d'ailleurs spontanément dans les entretiens qualitatifs). Il paraît donc légitime de distinguer, parmi les perceptions des inégalités, celles qui portent sur des biens sociaux de celles qui portent sur des discriminations. Il est possible que les attitudes à l'égard de ces deux types d'inégalités soient différentes et que les variables explicatives qui y sont associées le soient également.

Nous avons donc calculé deux types de scores concernant la perception de la force, de l'illégitimité et de l'impact personnel des inégalités. Le premier somme les réponses sur les inégalités de revenu et de patrimoine, que l'on qualifiera d'inégalités économiques, le second somme les réponses sur les inégalités ethniques, d'âge et de genre, que l'on qualifiera d'inégalités identitaires. On aurait pu intégrer les inégalités vis-à-vis des risques technologiques et de l'insécurité au score d'inégalités identitaires, mais les alphas de Cronbach étant très similaires, il nous a paru préférable de nous limiter aux discriminations communément évoquées dans le débat social: ethnie, genre, catégories d'âge. On remarquera que ces deux types d'inégalités renvoient à deux composantes de la position sociale que l'on a coutume de distinguer: les attributs que les sociologues qualifient habituellement d'achieved - statuts acquis comme le revenu ou la profession - et ceux qui sont qualifés d'ascribed - statuts assignés comme le sexe ou la génération. On pourra se demander s’il se dégage des liens particuliers entre ces deux composantes du statut et les deux types de perception des inégalités qui leur correspondent logiquement. 
Tableau I. Analyses en composantes principales sur les types d'inégalités

\begin{tabular}{|c|c|c|c|c|c|c|}
\hline \multirow[b]{2}{*}{ \% de variance expliquée } & \multicolumn{2}{|c|}{ FORCE } & \multicolumn{2}{|c|}{ ILLÉGITIMITÉ } & \multicolumn{2}{|c|}{ IMPACT PERSONNEL } \\
\hline & $39 \%$ & $10 \%$ & $58 \%$ & $10 \%$ & $34 \%$ & $9 \%$ \\
\hline facteurs: & $\mathrm{FI}$ & F2 & $\mathrm{FI}$ & F2 & $\mathrm{FI}$ & $\mathrm{F} 2$ \\
\hline LES INÉGALITÉS DE REVENU & 0,616 & 0,507 & 0,623 & 0,617 & 0,651 & 0,430 \\
\hline LES INÉGALITÉS DE PATRIMOINE & 0,458 & 0,673 & 0,493 & 0,738 & 0,530 & 0,532 \\
\hline $\begin{array}{l}\text { LES INÉGALITÉS FACE AU CHÔMAGE ET AUX } \\
\text { EMPLOIS PRÉCAIRES }\end{array}$ & 0,655 & 0,274 & 0,802 & 0,175 & 0,629 & 0,218 \\
\hline LES INÉGALITÉS DANS LES ÉTUDES SCOLAIRES & 0,644 & 0,066 & 0,789 & $-0,007$ & 0,522 & 0,052 \\
\hline LES INÉGALITÉS D'ACCÈS AUX SOINS MÉDICAUX & 0,615 & $-0,197$ & 0,803 & $-0,|4|$ & 0,589 & $-0,018$ \\
\hline $\begin{array}{l}\text { LES INÉGALITÉS D'EXPOSITION AUX RISQUES } \\
\text { TECHNOLOGIQUES }\end{array}$ & 0,600 & $\underline{-0,346}$ & 0,730 & $-0,065$ & 0,541 & $\underline{-0,202}$ \\
\hline LES INÉGALITÉS LIÉES À L'ORIGINE ETHNIQUE & 0,613 & 0,019 & 0,775 & $\underline{-0,203}$ & 0,573 & $\underline{-0,348}$ \\
\hline $\begin{array}{l}\text { LES INÉGALITÉS ENTRE LES HOMMES ET LES } \\
\text { FEMMES }\end{array}$ & 0,643 & $-0,262$ & 0,823 & $\underline{-0,254}$ & 0,531 & $\underline{-0,357}$ \\
\hline $\begin{array}{l}\text { LES INÉGALITÉS ENTRE LES JEUNES ET LES } \\
\text { PLUS ÂGÉS }\end{array}$ & 0,601 & $-0,336$ & 0,806 & $\underline{-0,199}$ & 0,602 & $\underline{-0,248}$ \\
\hline LES INÉGALITÉS FACE À L'INSÉCURITÉ & 0,609 & $\underline{-0,331}$ & 0,822 & $\underline{-0,201}$ & 0,600 & $-0,381$ \\
\hline LES INÉGALITÉS DE LOGEMENT & 0,721 & 0,040 & 0,842 & $-0,049$ & 0,680 & 0,145 \\
\hline LES INÉGALITÉS DE PÉNIBILITÉ DU TRAVAIL & 0,713 & 0,024 & 0,801 & 0,014 & 0,550 & 0,075 \\
\hline
\end{tabular}

Lecture: les corrélations positives au deuxième facteur sont en gras, les corrélations négatives en italique souligné

\section{LES THÉORIES EXPLICATIVES}

Nous ne chercherons pas à vérifier empiriquement la validité de l'ensemble des théories envisageables tout d’abord parce que les données dont nous disposons ne sont pas adaptées à l'évaluation de certaines d'entre elles. Par exemple, la théorie de «l'effet miroir » ne peut être testée qu'avec des données comparant la perception des inégalités dans plusieurs pays alors que notre corpus de données ne porte que sur la France. Au demeurant, cette théo-

7 Selon une telle théorie, le degré d'inégalité ressenti dans la société serait simplement le reflet de l'inégalité réelle, telle qu'elle est mesurée au niveau macrosocial (par le coefficient de Gini, par exemple). Les personnes, quel que soit leur statut, ressentiraient la société et les inégalités qui la traversent telles qu'elles sont effectivement. 
rie paraît bien fragile dans ce cas, puisque la France est sans doute un des pays où l'écart entre la mesure objective des inégalités et la mesure subjective de ces dernières est le plus élevé (OCDE, 2008; Forsé, Parodi, 2007). Nous n’avons pas non plus beaucoup d'éléments pour évaluer la théorie de «l’attribution interne» selon laquelle les individus se conduiraient comme des «psychologues naïfs » en attribuant leurs succès et leurs échecs à des causes internes (Heider, 1958 ; Ross, 1977 ; Stolte, 1983 ; et pour une discussion de ces théories, Shepelak, 1987). Cette hypothèse psychosociologique n'est pas facile à tester avec le matériel empirique dont nous disposons. Des méthodes expérimentales seraient sans doute mieux adaptées à son évaluation.

La théorie de légitimation de l'existant (Homans, 1974; Gijsberts, 2002) selon laquelle les personnes adapteraient leurs standards normatifs à leur perception de la situation peut également être écartée rapidement. En effet, les données descriptives que nous avons présentées dans la première partie de cet article montrent que les Français trouvent les inégalités à la fois fortes et condamnables : ils ne semblent donc pas du tout portés à justifier les inégalités en raison de leur présence même dans la société ; c'est plutôt l’inverse qui semble vrai.

Demeurent trois théories qui peuvent être testées avec notre corpus de données. La première est une théorie de la justification par l'intérêt bien compris. «Intérêt bien compris» ne renvoie pas ici à l'interprétation qu'en donne John Rawls (197I), qui s'intéresse aux choix que feraient des personnes placées sous un «voile d'ignorance», c'est-à-dire ne sachant pas quelle serait leur position personnelle dans les sociétés qu’elles doivent évaluer. Les personnes jugent alors des sociétés en intégrant l'idée que le sort pourrait les placer dans des positions peu favorables. Bien au contraire, nous nous intéressons ici à des personnes qui savent quelle est leur position. Les personnes situées au bas de la hiérarchie sociale seraient alors conduites à condamner plus souvent les inégalités, tout simplement parce qu'elles en sont elles-mêmes victimes. À l'inverse, les personnes ayant un statut élevé auraient tendance à justifier ces inégalités pour préserver la légitimité de leur position. En fait, cette idée de l'«intérêt bien compris» pourrait aussi être dénommée thèse de l'effet «position» (structural position thesis - Hadler, 2005) : les personnes différeraient dans leurs opinions sur 
l'inégalité en fonction de leurs positions sociales et des intérêts qui y sont associés. Les travaux anglo-saxons menés sur cette question semblent plutôt montrer une liaison assez régulière entre la position sociale et les attitudes à l'égard des inégalités (Kelley et Evans, 1993; Gijsberts, 2002). Mais ces travaux portent essentiellement sur la perception des inégalités de revenu et mettent surtout en lumière un effet du revenu du ménage. Par ailleurs, ils portent souvent sur le niveau de rémunération jugé «légitime» des hauts et des bas revenus et l'écart tolérable entre les deux (Kelley et Evans, 1993), ce qui est évidemment une perspective un peu différente de celle consistant à apprécier les inégalités de revenu en général (et encore plus l’ensemble des inégalités). Dans un travail plus récent fondé sur les données de l'enquête ISSP 1999-2000, Markus Hadler aboutit à un résultat inverse : les effets de la «stratification verticale», le revenu du ménage et les principales catégories de la définition de classe inspirées de Goldthorpe n'exerceraient pas d'effet significatif sur la perception des inégalités de rémunérations.

La deuxième théorie possible complète et nuance la théorie de la justification par l'intérêt bien compris. Elle repose en effet sur l'idée que l'intérêt en question ne peut être évalué exclusivement de manière absolue, mais peut être aussi considéré d'un point de vue relatif. Cette idée est issue de travaux sociologiques classiques sur la «frustration relative». Les sociologues ont montré depuis longtemps (Stouffer, 1949; Runciman, 1966) l'influence de ces mécanismes comparatifs sur la représentation des inégalités. La question du groupe de référence auquel les individus se comparent dans l'estimation de leur situation individuelle est essentielle pour forger leur sentiment d'iniquité. La «frustration» et le sentiment d'inégalité qui l'accompagne serait donc «relative »: tout dépendrait du groupe auquel les individus ont le sentiment d’appartenir (ou souhaitent appartenir) ou, comme l’a montré Raymond Boudon (1977), de leur position dans des structures d'interaction dans lesquelles ils sont en compétition pour un bien avec des chances variables de gain et de promotion. Il est tout à fait envisageable qu'un individu situé dans les franges inférieures de la stratification sociale n'en conçoive pas néanmoins de sentiment d'iniquité s'il se réfère d’abord dans ses comparaisons sociales aux membres de cette 
strate ou de ce groupe professionnel et que, par exemple, les rémunérations ou les promotions y soient régies par des règles égalitaires. À l'inverse, un individu situé dans des strates sociales plus élevées peut ressentir cette frustration s’il se compare à des collègues de même niveau qu'il estime favorisés.

Une troisième théorie possible ne repose plus, comme les précédentes, sur les liens supposés entre la position sociale occupée par les individus et les justifications qu'ils avancent pour interpréter et juger les inégalités. Elle suppose que ces justifications sont en grande partie indépendantes de la position occupée, ou en tout cas exercent un effet qui en est très largement indépendant. C'est donc une théorie de la justification par les valeurs. Cet effet idéologique peut être national: différentes sociétés se distinguent par une vision propre des inégalités (Huber et Form, 1973). Par exemple, les sociologues américains insistent sur l'éthique extrêmement individualiste qui prévaut dans leur pays pour expliquer le rejet des idées égalitaires qui y domine : selon cette éthique, les individus doivent assumer la complète responsabilité personnelle de leur fortune ou infortune (Della Fave, I974; Kluegel et Smith, 2009). À l'intérieur même d'une société, différentes conceptions coexistent et peuvent moduler le sentiment d'iniquité et la perception de l'inégalité sociale et des moyens de la corriger: ce que Della Fave (1974) appelle une «structure sous-jacente de croyance». On peut distinguer à cet égard au moins trois types d'orientation possibles: une orientation «égalitariste» qui valorise l'égalité comme une valeur per se, devant être respectée en toutes circonstances; une orientation «fonctionnaliste » qui considère au contraire l'inégalité comme nécessaire à la prospérité d’une nation ${ }^{8}$ et enfin une orientation «méritocratique» qui soutient que les efforts individuels sont le critère le plus important du succès et peuvent conduire à moduler les rétributions que reçoivent les individus.

8 Cette orientation «fonctionnaliste» peut aussi avoir un versant non économique, un certain nombre de personnes jugeant les inégalités inévitables car inhérentes à la nature humaine sans leur donner de justification économique. 


\section{LES QUESTIONS DE RECHERCHE}

Nous essaierons donc d'évaluer empiriquement les théories de l'intérêt bien compris, de la frustration relative et de l'effet des valeurs. Bien entendu, nous ne prétendons pas apporter de réponses définitives. Nos données sont limitées et nos variables ne sont pas toujours parfaitement adaptées aux questions théoriques que nous posons. Précisément, comment construire des indicateurs qui permettent de reformuler ces différentes théories en termes évaluables par notre enquête (avec les limites que cela suppose)?

Si la théorie de l'intérêt bien compris est vérifiée, on peut supposer trouver une forte liaison entre la position sociale occupée par les individus et leur perception des inégalités, dans le sens suivant: plus les individus occupent une position élevée dans la stratification sociale, plus ils s’accommoderaient des inégalités dans la société. Il reste à déterminer de quels types d’inégalités il sagit (accès aux biens sociaux ou discriminations) et comment l'on définit la position sociale.

Ce dernier point est très important car une approche restrictive de la position sociale, limitant l'analyse aux seuls attributs socio-économiques, paraîtrait mal adaptée à notre sujet puisque nous avons pris en compte une gamme très large d’inégalités relevant, pour les unes de l'accès aux biens sociaux, pour les autres des discriminations. Une approche large de la position sociale ${ }^{9}$ permet ainsi d'enrichir l'analyse et la vérification de nos hypothèses. Par exemple, si la théorie de l'intérêt bien compris est vérifiée, les différentes composantes de la position sociale devraient être associées de manière particulière à la perception des différents types d'inégalités :

- les riches ou les PCS élevées devraient être plus accommodants avec les inégalités économiques, mais il n'y a pas de raison (si l'on s'en tient à cette théorie) qu'ils le soient avec les discriminations ;

- les personnes potentiellement victimes de discriminations - jeunes, femmes, étrangers notamment - devraient y être plus sensibles.

9 Elle découle des travaux de Peter Blau et renvoie au jeu complet des variables de la signalétique usuelle dans les enquêtes. Aux éléments de la stratification socio-économique, il faut ajouter genre, âge/génération, ethnie, urbanisation du lieu de résidence. Les «positions sociales» sont alors définies par la combinatoire complète de tous ces éléments. 
Une vérification, au moins partielle, de la théorie de l'intérêt bien compris devrait donc trouver une liaison entre les attributs acquis (revenu, profession, niveau d'études) et la sensibilité aux inégalités d’accès aux biens sociaux, ainsi qu’une liaison entre les attributs assignés (sexe, âge, origine ethnique) et les discriminations.

On cherchera à apprécier la validité de la théorie de la frustration relative à travers un certain nombre de questions de l'enquête permettant d'établir une typologie des mécanismes de comparaison sociale dans la légitimité perçue de sa rémunération. On demandait en effet aux personnes interrogées d'indiquer si le montant de leur rémunération leur paraissait juste et d'indiquer ensuite sur quels critères elles se basaient pour dire que leur rémunération était ou n’était pas équitable (compétence, efforts, comparaison avec différentes catégories).

La recherche d'une validation de l'effet des valeurs sur la perception des inégalités est limitée par les variables disponibles. En effet, les questions qui ont été posées à ce sujet ne portent que sur la justification (ou la condamnation) des inégalités de revenu (attachement à l'égalité, à la méritocratie, croyance dans le caractère inévitable des inégalités pour le fonctionnement de l'économie, etc.). Le volet «discriminations » des inégalités et les orientations de valeurs qui pourraient lui être associées ne peut donc être examiné avec nos données: on aurait pu par exemple supposer que les attitudes à l'égard des discriminations étaient associées au degré d’adhésion aux valeurs $\left\langle\right.$ universalistes $\gg^{10}$ telles qu'elles sont définies par Gérard Grunberg et Étienne Schweisguth (1997). Les questions dont nous disposons nous permettent cependant de couvrir le champ très important des principes de justice en matière d'inégalités économiques qui renvoient d’ailleurs à des orientations beaucoup plus larges concernant l'égalité et les modulations qu’on peut lui apporter.

Ces trois théories explicatives ne sont évidemment pas indépendantes les unes des autres et une des questions centrales sera de tenter d'établir comment elles s'articulent. C’est l'hypothèse que voulait déjà explorer l'enquête précédente du GEMAS (1976) : «les conceptions que les individus expriment sur ce que devrait être la justice sociale sont moins déterminées par la situation objective des inté-

I0 Selon ces auteurs, les valeurs universalistes prônent l'égale dignité de tout être humain quelle que soient son origine et sa condition. Un bon moyen de les tester aurait été d'examiner les attitudes à l'égard des immigrés si nous avions disposé de questions sur ce sujet. 
ressés que par la perception qu'ils ont des inégalités existantes ». Un des enjeux fondamentaux de la recherche est donc de tenter d'évaluer à quel degré les individus évaluent les inégalités globales en fonction de leur position dans la stratification sociale et à quel point ils le font en fonction de principes de justice indépendants de cette position. Il est probable que l'opinion courante admette que les individus expriment des opinions sur les inégalités dans la société très dépendantes de leur propre position sociale: c'est par exemple l'image traditionnelle d'une société de «classes» opposant des groupes sociaux aux intérêts divergents et ayant conscience de ces intérêts. Nous verrons que cette vision ne résiste pas à nos données.

\section{LES RÉSULTATS}

Nous examinerons successivement l'effet de la position sociale, l'effet des mécanismes de comparaison sociale et, enfin, l'effet des valeurs justifiant ou non les inégalités économiques.

\section{I. L'EFFET DE LA POSITION SOCIALE}

L'examen de l'impact des différentes composantes de la position sociale sur la perception des inégalités (Tableau 2), ne plaide pas, à première vue, en faveur de la thèse de l'intérêt bien compris. En effet, s'il y a un lien net entre la position sociale et le fait de se sentir personnellement affecté ou non par les inégalités ( $28 \%$ de la variance est expliquée par les variables de position sociale), ce lien est beaucoup plus faible lorsqu'on examine les relations de cette position sociale avec la perception des inégalités sociales globales (force et illégitimité). Le modèle de «force des inégalités » est certes statistiquement significatif (au seuil de I \%) mais la part de variance expliquée est faible (5\%). Cette part est encore plus faible pour le modèle «d'illégitimité » $(3 \%)$ et le modèle n’atteint pas le seuil de significativité (au seuil de $5 \%$ ). On peut penser que les personnes ne transposent pas leur situation personnelle à l'analyse de la société globale : ceux qui se sentent personnellement défavorisés le sont dans une large mesure objectivement, comme sont favorisés ceux qui le ressentent subjectivement, mais ces différents groupes de Français, affectés très différemment par les inégalités, se retrouvent sur une position relativement proche 
pour analyser l'état et la légitimité des inégalités dans la société. De fait, l'introduction du sentiment d'être personnellement touché par les inégalités (sous la forme d'une variable discrète découpée en quintiles) comme variable explicative supplémentaire n’améliore quassez faiblement le pouvoir explicatif du modèle de perception de la «force des inégalités» (le $\mathrm{R}^{2}$ passe de 5 à $7 \%$ ) et pas du tout le pouvoir explicatif du modèle expliquant l'opinion sur «l'illégitimité ». Seuls les Français qui se sentent très fortement touchés par les inégalités, ceux qui appartiennent au dernier quintile, considèrent les inégalités sociales globales comme particulièrement fortes. Ces personnes très insatisfaites de leur situation personnelle se trouvent dans toutes les catégories de la population mais sont plus fortement représentées dans les catégories qui cumulent faibles revenus et faible niveau d'études. Au total, en dépit de ces nuances, ce n’est pas principalement la situation personnelle qui informe la perception globale.

Tableau 2. L’effet de la position sociale sur l'impact personnel, la force et l'illégitimité ressentis des inégalités

\begin{tabular}{|c|c|c|c|}
\hline & \multicolumn{3}{|c|}{ VARIANCE PAR DEGRÉ DE LIBERTÉ } \\
\hline & IMPACT PERSONNEL & FORCE & ILLÉGITIMITÉ \\
\hline SEXE & - & $9,3 * * * *$ & $3,0 *$ \\
\hline$\hat{A} G E$ & 4,1 米米米 & $4,3 * * * *$ & - \\
\hline ORIGINE ETHNIQUE & 5,9 ***** & - & $4,3 * * * *$ \\
\hline URBANISATION & $7,5 * * * *$ & - & - \\
\hline TYPE D'ACTIVITÉ & $6,0 * * * *$ & - & I,7* \\
\hline PCS & $1,2 *$ & I,7*** & - \\
\hline NIVEAU D'ÉDUCATION & |5,|米米米 & - & - \\
\hline REVENU & 19,3 ***** & - & \\
\hline $\mathrm{R}^{2}$ & 0,28 & 0,05 & 0,03 \\
\hline
\end{tabular}

Lecture: Analyse de variance des scores de force ressentie des inégalités, d'illégitimité et d'impact personnel, contrôlés par toutes les variables du tableau; ****: sig. au seuil de 1\%, **: au seuil de $5 \%$ * : au seuil de 10\%; les cases vides sont non significatives. Les scores sont des valeurs centrées réduites de moyenne 0 (variant de -3,8 à 2,4 pour le score de force des inégalités; de -3,7 à 1,5 pour le score d'illégitimité; de -2,6 à 3,9 pour le score d'impact personnel) 
Le tableau 2 porte néanmoins sur des indicateurs agrégés de perception des inégalités. Or, nous l'avons vu, il y a deux types différents d’inégalités, des inégalités économiques et des inégalités qui relèvent plutôt de discriminations. Aussi l'effet position pourrait être en partie masqué par la seule prise en compte de l'indicateur agrégé, si des liaisons spécifiques se manifestaient d'une part entre les composantes acquises de la position sociale et la sensibilité aux inégalités économiques, et d’autre part entre les composantes assignées et la sensibilité aux discriminations. Ces liaisons spécifiques se manifestent-elles et renforcent-elles ainsi la thèse de l'intérêt bien compris ?

La réponse, issue du tableau 3, est en partie positive, mais en partie seulement. Elle est positive car il y a bien des liens entre certains attributs acquis de la position sociale et la sensibilité aux inégalités économiques dans l'ensemble de la société : il apparaît notamment que les personnes aux revenus et au niveau d'études élevés jugent les inégalités économiques plus tolérables que les personnes aux niveaux de revenu et d'étude plus faibles. Ce résultat est bien compatible avec la thèse de l'intérêt bien compris : les riches justifient plus facilement des inégalités économiques présentes dans la société que les pauvres. Ce résultat est d’autant plus significatif qu'il ne résulte pas d'un biais de perception : les riches ont même tendance à trouver les inégalités économiques plus présentes dans la société que les pauvres ; néanmoins ils les trouvent plus acceptables. 
Tableau 3. L’effet de la position sociale sur la perception des inégalités économiques et identitaires

\begin{tabular}{|c|c|c|c|c|}
\hline & \multicolumn{4}{|c|}{ VARIANCE PAR DEGRÉ DE LIBERTÉ } \\
\hline & \multicolumn{2}{|c|}{ FORCE DES INÉGALITÉS } & \multicolumn{2}{|c|}{ ILLÉGITIMITÉ } \\
\hline & ÉCONOMIQUES & IDENTITAIRES & ÉCONOMIQUES & IDENTITAIRES \\
\hline SEXE & - & |4,07**** & - & $5,98 * * * *$ \\
\hline$\hat{A} G E$ & 3,76 **** & - & $2,90 * *$ & - \\
\hline ORIGINE ETHNIQUE & - & - & - & $3,28 * *$ \\
\hline URBANISATION & $2,79 * * * *$ & - & - & - \\
\hline TYPE D'ACTIVITÉ & - & $1,92 *$ & - & - \\
\hline PCS & - & - & 1,98*** & - \\
\hline NIVEAU D'ÉTUDES & 4,93**** & - & $2,37 *$ & $4,66 * * * * *$ \\
\hline REVENU & $|, 8|^{*}$ & - & 4,36 **** & - \\
\hline $\mathrm{R}^{2}$ & 0,05 & 0,04 & 0,06 & 0,03 \\
\hline
\end{tabular}

Lecture: voirTableau 2

Des liens apparaissent également entre certaines composantes assignées de la position sociale et la sensibilité aux discriminations : c'est le cas notamment du sexe, les femmes jugeant les discriminations plus inacceptables que les hommes, alors qu’elles ont la même sensibilité aux inégalités économiques. Cette sensibilité féminine aux discriminations apparaît bien également dans les entretiens qui confirment une forte sensibilité féminine à l'injustice faite aux femmes dans la vie économique : ce qui ressort c'est le sentiment de discrimination sur le plan de la rémunération mais aussi d'une manière plus générale, la discrimination ressentie par ces femmes en comparaison avec des hommes de même niveau d'études. L'origine ethnique est également associée à la sensibilité aux discriminations : ce ne sont pas cependant les personnes d'origine maghrébine qui y sont le plus sensibles, mais celles d'une autre origine étrangère.

Ces résultats redonnent donc un peu de poids à la thèse de «l'effet position», sans pourtant la renforcer très fortement car la variation des perceptions des inégalités économiques comme des inégalités identitaires ne reste que très faiblement expliquée par les différentes variables de position (voir les $\mathrm{R}^{2}$ du tableau 3). 
Par ailleurs, certaines liaisons ne semblent pas rentrer dans ce cadre explicatif: on constate notamment que les personnes ayant un niveau d'études élevé condamnent plus souvent les discriminations que celles qui ont un niveau d'études plus faible. Ce résultat s'explique très probablement par le fait que l’adhésion aux valeurs universalistes est très corrélée au niveau d'études, les personnes ayant fait des études supérieures y adhérant beaucoup plus fortement que les autres. Par ailleurs, l'âge n’a pas d'effet significatif sur la sensibilité aux discriminations : les jeunes, par exemple, n'y sont pas plus sensibles que les autres classes d'âge.

Même en prenant en compte la distinction entre inégalités économiques et inégalités identitaires et en les mettant en relation avec les composantes acquises et assignées de la position sociale, on constate donc que ces relations ne jouent pas toujours dans le sens attendu et que l'effet statistique de la position sociale sur la perception des inégalités reste faible.

Cependant, ou pourrait penser que les appréciations sur les inégalités étudiées jusqu'à présent sont très générales et qu’elles différent peut-être des opinions des Français sur les revenus des différentes professions et des correctifs qu'il faudrait éventuellement y apporter. C'est une autre façon, plus directement reliée à la vie sociale réelle, de juger de leurs perceptions à ce sujet. L'enquête PISJ permet de les évaluer puisqu'elle posait des questions sur les revenus perçus et souhaités par les personnes interrogées pour différentes professions. En calculant le rapport entre ces deux revenus on peut ainsi avoir une mesure de l'ampleur des corrections souhaitées pour une profession donnée.

L'examen des variations en fonction du niveau de revenu du ménage pour deux professions situées aux extrémités de la pyramide sociale, les ouvriers non qualifiés et les PDG, semble montrer un large consensus sur les correctifs à apporter aux rémunérations de ces deux professions quel que soit le niveau de revenu du ménage auquel la personne appartient: tous les Français, quel que soit leur niveau d'aisance (et de diplôme), sont d’accord pour considérer que les PDG sont sur-rémunérés et que les ouvriers non qualifiés sont sous-rémunérés et l'appréciation du niveau de correction souhaitable de cette sur ou sous-rémunération varie très peu selon la situation financière ou éducative des personnes interrogées. Certes, la rémunération souhaitée pour les PDG croît bien avec le niveau de revenu 
du ménage auquel appartient la personne interrogée." Mais comme leur revenu estimé est également jugé plus élevé par les Français les plus aisés, le correctif apporté reste à peu près constant (et élevé) quel que soit le revenu (Figure 2)

Figure 2. Rémunération estimée et souhaitée pour les PDG selon le niveau de revenu du ménage

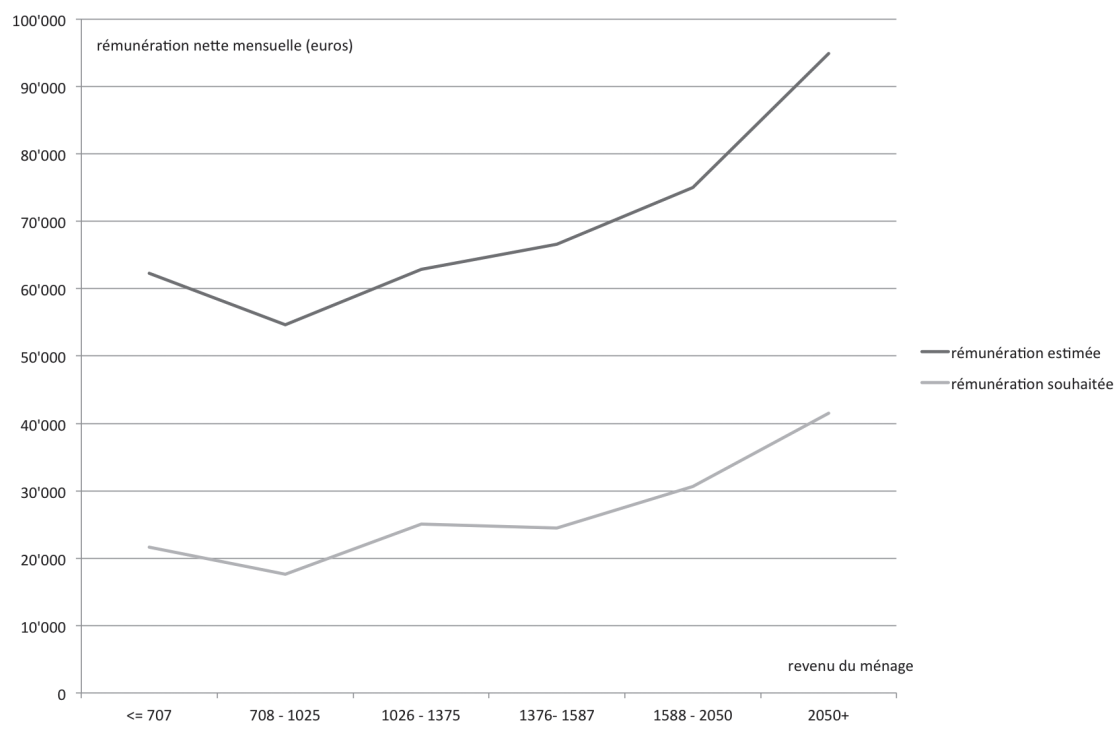

L’examen des évaluations plus factuelles sur les écarts estimés et souhaités de rémunérations des professions ne contribue donc pas non plus à renforcer la thèse de l'intérêt bien compris. Au total, elle ne peut certainement pas prétendre constituer l'explication principale de la variation des perceptions au sujet des inégalités sociales.

\subsection{L'EFFET DES MÉCANISMES DE COMPARAISON SOCIALE}

Si les entretiens qualitatifs semblent montrer que les mécanismes de comparaison jouent un grand rôle dans la formation des opinions au sujet des inégalités, ils ne jouent pas cependant de manière systématique. Certaines des personnes interrogées ne se comparent pas à d’autres et se contentent de 
juger de façon intrinsèque leur situation par rapport à des aspirations individuelles. Si ces aspirations - par exemple une forme de réussite professionnelle correspondant aux attentes initiales - sont satisfaites, les inégalités qui pourraient être ressenties passent au second plan ou sont même acceptées comme la conséquence d'un choix personnel. Cet état d'esprit conduit donc probablement à atténuer la sensibilité aux inégalités.

La comparaison avec d'autres peut au contraire la stimuler. Mais c'est loin d'être toujours le cas, notamment lorsque le groupe de référence est le groupe d’appartenance. Les interviews montrent en effet que ceux qui se comparent aux membres de leur univers professionnel, aux «collègues », ressentent un faible sentiment d'injustice lorsque cette comparaison se fait à l'intérieur d'un groupe régi par des normes égalitaires. On retrouve là un thème classique des mécanismes de frustration relative mis en lumière par la recherche bien connue de l'American Soldier: Stouffer (1949) montre que les membres des corps où la promotion est rare, s'estiment satisfaits du système de promotion. C'est donc moins la récompense du mérite en tant que telle qui importe, que la comparaison avec les collègues de même niveau. Dans les interviews réalisées, il n’y a pas d’insatisfaction exprimée par ceux qui connaissent ce type de rémunération fondé sur des principes d’ancienneté, qui serait liée à l’absence de récompense du «mérite ». Bien sûr, ces comparaisons peuvent aussi, dans d'autres contextes de travail, être sources d'insatisfaction et de frustration. Dans les entretiens ce sont surtout des femmes qui expriment les sentiments les plus nets à ce sujet, lorsqu'elles s'estiment discriminées par rapport à des collègues masculins de même niveau professionnel et mieux rémunérés. Dans ce cas, leur groupe de référence est celui des collègues masculins mieux payés.

Un des enseignements importants des entretiens est donc que parmi les personnes satisfaites de leur situation, il faut distinguer celles qui le sont parce qu'elles refusent toute comparaison, et celles qui le sont parce qu'elles tirent un bilan positif de leur comparaison à d'autres catégories sociales. De la même manière on peut supposer que, parmi les personnes insatisfaites, certaines le sont à la suite de comparaisons négatives, alors que d'autres ne se fondent que sur des critères personnels (la prise en compte de leur valeur professionnelle intrinsèque). 
Pour tenter d'opérationnaliser cette idée, nous sommes partis de deux séries de questions de l'enquête PISJ : une question sur l'appréciation par les personnes interrogées de la justice de leur rémunération; une question sur les critères sur lesquels elles s'appuient pour dire que leur rémunération est ou n'est pas équitable. Parmi ces critères, on proposait aux personnes interviewées des réponses ayant trait à leur compétence ou à leurs efforts, et d’autres mettant explicitement en avant la comparaison avec d’autres catégories sociales. Nous avons donc retenu comme approximation d'une situation possible de frustration relative les cas où les personnes s'estiment sous-rémunérées en fondant ce jugement sur la comparaison avec d'autres catégories sociales. Il y a dans ces cas de bonnes raisons de penser que le groupe de référence pour la personne en question est bien celui qui sert de support à cette comparaison négative. À partir de ces deux questions, une variable à quatre modalités a donc été créée :

1. Les satisfaits intrinsèques estiment être rémunérés justement ou plus haut que ce qui est juste et ne se comparent pas à d'autres, ou n'attachent qu'une importance limitée à cette comparaison (en se situant à un rang inférieur à 5 sur l'échelle de 1 pas important du tout à 10 très important) (7\% de la population ayant déjà exercé un emploi).

2. Les satisfaits relatifs estiment être rémunérés justement ou plus haut que ce qui est juste, et attachent de l'importance à la comparaison avec d'autres (en se situant sur un rang supérieur à 5), qu'il s’agisse de collègues, de gens du même âge, de la moyenne des Français, et des personnes les mieux rémunérées de leur entreprise ou de leur secteur (29\%).

3. Les insatisfaits intrinsèques estiment être rémunérés injustement (un peu ou beaucoup), mais sans se comparer à d'autres (13\%).

4. Les insatisfaits relatifs estiment être rémunérés injustement en se comparant à d'autres (51\%).

L'introduction de cette variable dans les modèles statistiques considérés antérieurement n’améliore pas substantiellement leur capacité prédictive. ${ }^{12}$ Globalement, la «frustration relative» ne semble pas être un mécanisme puissant de sensibilisation aux inégalités sociales globales. Cependant, la variable 
elle-même exerce un effet significatif pour expliquer la perception de la force des inégalités sociales (pas de leur illégitimité cependant). Si cet effet n’améliore pas le pouvoir explicatif de l'ensemble des variables prises en compte, c'est qu'il s'exerce en affaiblissant en même temps la variance expliquée par deux autres variables : l'âge et la PCS. Il s'avère en effet que la «frustration relative » est associée à des effets de cycle de vie et de catégorie sociale : les «insatisfaits relatifs » sont notamment plus présents au milieu du cycle de vie, et ils sont plus représentés également dans les classes moyennes et classes moyennes inférieures (professions intermédiaires, ouvriers et employés qualifiés). Cependant même si l'effet des mécanismes de comparaison sociale sur la perception des inégalités dans la société est limité, il est statistiquement significatif et plus important que l'effet de la position sociale stricto sensu.

Cet effet des mécanismes de comparaison sociale sur la force perçue des inégalités dans la société fonctionne dans le sens attendu par les résultats des entretiens qualitatifs (Figure 3) : selon qu'elle est ou non associée à des comparaisons avec d'autres catégories sociales, la satisfaction ou l'insatisfaction à l'égard de sa rémunération personnelle débouche sur une perception plus ou moins forte des inégalités sociales globales. Il y a bien notamment un effet spécifique de la «frustration relative » : ceux qui se sentent sous-rémunérés en se comparant à d'autres ont une sensibilité plus forte aux inégalités sociales que les personnes satisfaites de leur sort bien sûr; mais ils ressentent également ces inégalités plus fortement que les personnes qui jugent leur rémunération insuffisante pour des raisons intrinsèques indépendantes de ce jeu de comparaison sociale. 
Figure 3. Effet «toutes choses égales par ailleurs» de la «frustration relative» sur la perception des inégalités sociales globales

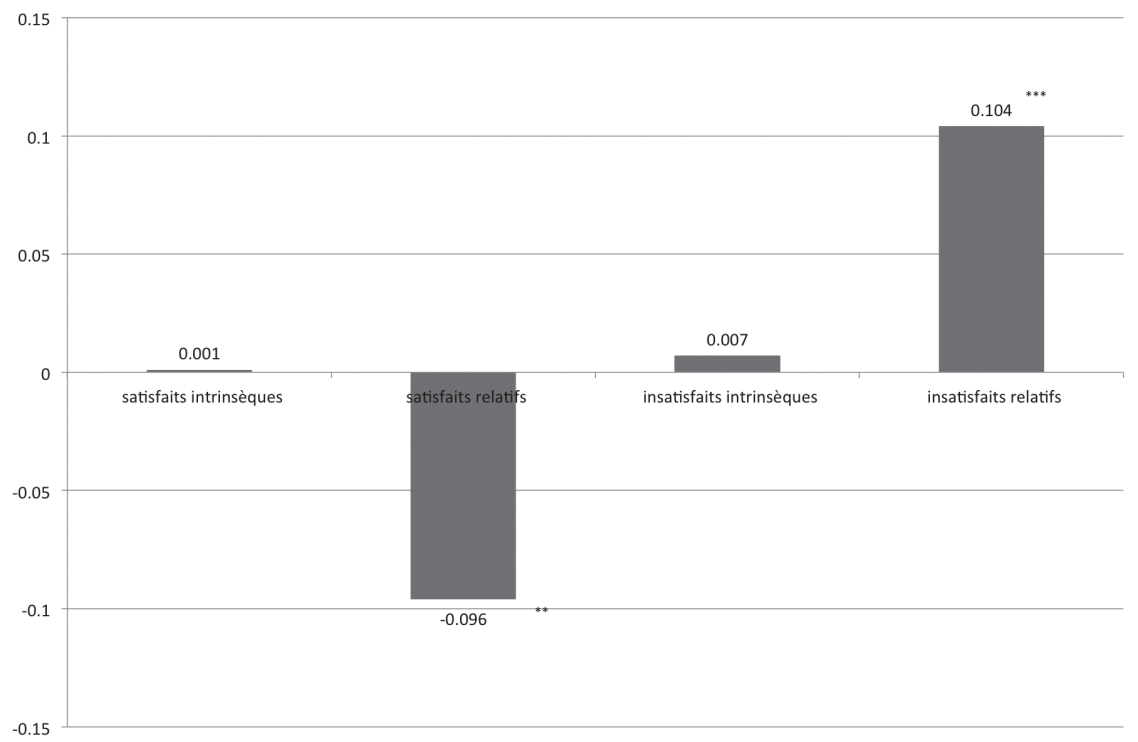

Lecture: valeurs des coefficients d'une analyse de variance sur le score de force estimée des inégalités (valeurs centrées-réduites, de moyenne 0 et d'écart-type I pour l'ensemble de la population) en fonction des modalités de frustration relative et en tenant compte du sexe, de l'âge, du type d'activité, de la profession, du niveau d'études, de l'origine ethnique, du lieu de résidence et du revenu du ménage. ****: sign. au seuil de $1 \%$.

*** sign. au seuil de $5 \%$.

\subsection{L'EFFET DES VALEURS}

Les principes de justice et la perception des inégalités

Les enquêtes internationales montrent que la représentation de la structure sociale et des inégalités peut varier fortement d'un pays à l’autre (Forsé, Parodi, 2007). Les Français ont une représentation de la pyramide sociale très hiérarchisée avec une base très large et une élite très restreinte. Ils sont aussi très nombreux à estimer que «les inégalités de revenu dans le pays sont trop grandes» (60\% sont tout à fait d'accord avec cette idée contre, par exemple, 25\% des Nord-Américains). Ils 
perçoivent donc, plus que dans d’autres pays, la société comme très inégalitaire et sont largement et fortement convaincus qu'il faut corriger cet état de fait.

Nous avons vu que les opinions sur l'état des inégalités dans la société française - leur importance, leur acceptabilité - ne sont que très faiblement influencées par la position qu'occupent les individus dans la stratification sociale. Il semble donc que la plupart d'entre eux ne forment pas leur jugement en se fondant sur une rationalité de type instrumental qui viserait à justifier ou contester leur statut social personnel, selon qu'il est favorable ou défavorable. Se fonde-t-il alors sur des valeurs en partie déconnectées de ce statut?

Les informations sur les orientations de valeurs des personnes ne sont malheureusement pas très nombreuses dans l'enquête PISJ. Nous disposons cependant d'une batterie de questions sur les motifs qui peuvent ou non conduire les Français à justifier un certain degré d’inégalité concernant les revenus.

Une analyse typologique réalisée à partir des premières coordonnées d'une analyse factorielle préalable, portant sur ces principes (la récompense du mérite ou des efforts dans le travail, la reconnaissance de la valeur du diplôme, l'attachement par principe à l'égalité ou la conviction que les inégalités sont inévitables pour qu’une économie soit dynamique, etc.) aboutit à la construction de cinq classes :

1. Les fonctionnalistes attachés à la méritocratie scolaire (31\% de la population) pensent que les inégalités sont économiquement nécessaires mais doivent être liées au diplôme.

2. Les fonctionnalistes attachés à la méritocratie des efforts personnels (13\%) partagent avec les premiers la conviction de la nécessité économique des inégalités mais pensent qu'elles doivent plutôt reposer sur la récompense des efforts individuels.

3. Les égalitaristes de principe (10\%) valorisent le principe d'égalité en tant que tel.

4. Les tenants de l'équité (25\%) veulent réduire les inégalités par respect de la dignité personnelle tout en prenant en compte les efforts individuels.

5. Les «omnicritères 》 (21\%) veulent tenir compte à la fois des résultats dans le travail, des capacités et du diplôme. 
Quels sont les positionnements sociaux de ces cinq types? Globalement, les individus situés dans les strates supérieures de la stratification sociale sont plus éloignés des principes égalitaires et plus attachés à la méritocratie scolaire, tandis que les individus situés dans les strates inférieures sont plus attachés à un modèle égalitaire (plutôt les employés) ou à un modèle d'équité par la récompense des efforts, des résultats et des capacités non scolaires (plutôt les ouvriers) (Tableau 4).

Cependant ces caractéristiques ne semblent pas dessiner des types sociaux très tranchés: par exemple, les cadres sont présents à un niveau significatif dans tous les types, même s'ils sont un peu surreprésentés dans le type antiégalitariste; il en va de même pour les ouvriers et les personnels de service qui sont presque autant représentés que les cols blancs dans les types antiégalitaristes. Au total, la position sociale est finalement peu prédictive du type auquel on appartient, même si toutes les composantes de cette position (à l'exception de l'âge et de la catégorie socioprofessionnelle lorsqu'on prend en compte simultanément le niveau d'études et le niveau de revenu) ont des effets significativement différents de zéro: le pseudo-R² de McFadden d'une régression logistique multimoniale est de 0,04I (il varie entre o et I pour un modèle parfait). Le pouvoir explicatif des justifications des inégalités économiques par les différentes composantes de la position sociale est donc faible. 
Tableau 4. Poids des types d'orientation idéologique à l'égard des inégalités économiques dans chaque catégorie socioprofessionnelle

\begin{tabular}{|c|c|c|c|c|c|c|}
\hline & $\begin{array}{c}\text { FONCTIONNALISTES } \\
\text { ATTACHÉS } \\
\text { AU DIPLÔME }\end{array}$ & $\begin{array}{l}\text { FONCTIONNALISTES } \\
\text { ATTACHÉS AUX } \\
\text { EFFORTS }\end{array}$ & ÉGALITARISTES & $\begin{array}{l}\text { ÉQUITÉ } \\
\text { LLÉE AUX } \\
\text { EFFORTS }\end{array}$ & OMNICRITĖRES & \\
\hline AGRICULTEURS & 34,0 & 8,5 & 12,8 & 21,3 & 23,4 & 100,0 \\
\hline $\begin{array}{l}\text { INDUSTRIELS } \\
\text { GROS } \\
\text { COMMERÇANTS }\end{array}$ & & 75,0 & & 12,5 & 12,5 & 100,0 \\
\hline $\begin{array}{l}\text { ARTISANS, } \\
\text { PETITS } \\
\text { COMMERÇANTS }\end{array}$ & 27,3 & 18,2 & 5,2 & 20,8 & 28,6 & 100,0 \\
\hline $\begin{array}{l}\text { CADRES, PROF. } \\
\text { LIBÉRALES }\end{array}$ & 33,6 & 15,0 & 9,5 & 20,0 & 21,8 & 100,0 \\
\hline $\begin{array}{l}\text { PROF. } \\
\text { INTERMÉDIAIRES }\end{array}$ & 32,9 & 13,2 & 8,9 & 28,3 & 16,8 & 100,0 \\
\hline EMPLOYÉS & 30,1 & 10,6 & 14,0 & 24,0 & 21,2 & 100,0 \\
\hline $\begin{array}{l}\text { OUVRIERS } \\
\text { QUALIFIÉS }\end{array}$ & 25,3 & 16,2 & 5,2 & 31,4 & 21,8 & 100,0 \\
\hline $\begin{array}{l}\text { OUVRIERS NON } \\
\text { QUALIFIÉS }\end{array}$ & 26,3 & 8,8 & 11,4 & 28,9 & 24,6 & 100,0 \\
\hline $\begin{array}{l}\text { PERSONNEL DE } \\
\text { SERVICE }\end{array}$ & 35,0 & 3,9 & 8,7 & 27,2 & 25,2 & 100,0 \\
\hline $\begin{array}{l}\text { INACTIFS } \\
\text { (ÉTUDIANTS, } \\
\text { F. AU FOYER) }\end{array}$ & 31,5 & 12,6 & 13,2 & 22,7 & 19,9 & 100,0 \\
\hline TOTAL & 30,6 & 12,8 & 10,2 & 25,2 & 21,2 & 100,0 \\
\hline
\end{tabular}

La question principale qui nous intéresse vise à déterminer à quel point ces principes de justice sont associés aux perceptions des Français sur l’ampleur des inégalités dans la société et sur le degré auquel ils se sentent personnellement affectés par celles-ci. 
Cet effet est indéniable concernant la perception des inégalités sociales globales (Tableau 5): lorsqu'on prend en compte cette variable d'orientation idéologique, la part de variance expliquée est doublée par rapport à un modèle initial qui ne comportait comme variables indépendantes que les variables de position sociale des tableaux précédents. ${ }^{13}$ À l'inverse, la prise en compte des principes de justice n'a aucun effet sur la perception de l'impact personnel des inégalités sociales.

Tableau 5. Part de variance expliquée des scores de perception des inégalités, selon la prise en compte des attitudes de justification des inégalités économiques et du positionnement politique

\begin{tabular}{|l|c|c|c|}
\hline \multirow{2}{*}{} & \multicolumn{3}{|c|}{ INEGALITÉS RESSENTIES } \\
\cline { 2 - 4 } & FORCE & ILLÉGITIMITÉ & IMPACT PERSONNEL \\
\hline$R^{2}$ POSITION SOCIALE & 0,05 & 0,03 & 0,28 \\
\hline $\begin{array}{l}R^{2} \text { POSITION SOCIALE + «PRINCIPES DE } \\
\text { JUSTICE» }\end{array}$ & 0,10 & 0,06 & 0,28 \\
\hline $\begin{array}{l}R^{2} \text { POSITION SOCIALE + «PRINCIPES DE } \\
\text { JUSTICE + POSITIONNEMENT POLITIQUE }\end{array}$ & 0,12 & 0,06 & 0,29 \\
\hline
\end{tabular}

Lecture: Le $R^{2}$ mesure la part de variance expliquée par les variables de position sociale des tableaux précédents, sans la variable des principes de justice (I le ligne), ou avec la variable des principes de justice ( $2^{e}$ ligne), ou encore avec cette demière variable et la variable de positionnement sur l'échelle gauche-droite.

La prise en compte du positionnement politique, en sus des principes de justice, améliore encore le pouvoir explicatif d'ensemble du modèle d’analyse de la perception de la force des inégalités dans la société; mais c’est la première variable qui a l'effet le plus net: $36 \%$ de la variance expliquée ${ }^{14}$ par le modèle est imputable à cette variable idéologique, contre $20 \%$ à la variable de positionnement politique. À l'inverse, le positionnement politique n’a qu'un effet nul (illégitimité) ou faible (impact personnel) sur les autres aspects de la perception des inégalités.

13 Bien sûr, il n'est pas surprenant que le degré d'«illégitimité» ressenti à l'égard des inégalités sociales globales soit lié aux principes de justice auxquels adhèrent les individus. Mais on aurait pu s'attendre précisément à ce que l'introduction de cette variable améliore très fortement le pouvoir explicatif du modèle statistique de ce type de perception. Ce n'est pas vraiment le cas puisque le $\mathrm{R}^{2}$ du modèle illégitimité passe de 0,03 à 0,06 (deuxième colonne du tableau 5).

14 Variance expliquée, dont la part est elle-même de 12\%, on le rappelle. 
Le clivage se fait entre personnes à orientation «fonctionnaliste» et celles qui sont attachées à l'équité ou à des principes égalitaires. Quelle que soit leur position sociale, les premières trouvent les inégalités globales moins fortes et moins injustifiées que les secondes (Figure 4). Il ne s'agit pas d’une divergence sur les seules inégalités économiques, que les «fonctionnalistes» trouveraient logiquement plus tolérables, mais bien d'une différence générale: toute inégalité, quelle que soit son type, est jugée par eux moins forte et plus tolérable. Lampleur de l'écart mérite d'être soulignée: il est, par exemple, beaucoup plus important que ne le sont les écarts suivant le niveau de diplôme. La conséquence en est que les $\mathrm{R}^{2}$ des analyses de variance sur les jugements à propos de la société globale passent de 0,05 à o, IO (jugement sur l’ampleur des inégalités) et de o,03 à o,06 (jugement sur l'illégitimité des inégalités) quand on ajoute à cette position sociale l'orientation en matière de justice sociale.

Figure 4. Effets des principes de justice sur la perception des inégalités sociales (effets nets à position sociale contrôlée)

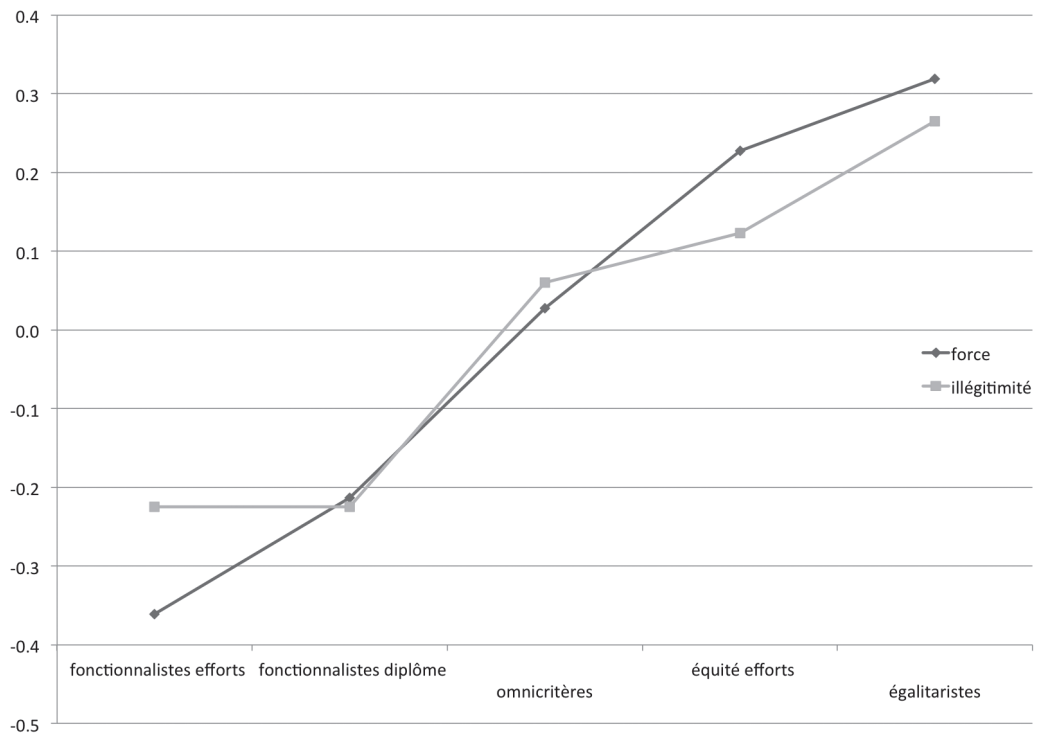

Lecture: variation des scores de force estimée et d'illégitimité des inégalités (valeurs centréesréduites, de moyenne 0 et d'écart-type I pour l'ensemble de la population) en fonction des principes de justice et en tenant compte du sexe, de l'âge, du statut d'activité, de la profession, du niveau d'études, du revenu du ménage, de l'origine ethnique et du type d'habitat. 
Il est frappant en contraste de constater la faible relation entre ces orientations en matière de justice et l'impact personnellement ressenti des inégalités. Condamner ou non le principe même des inégalités, légitimer la prise en compte du mérite individuel dans les rétributions personnelles, considérer les inégalités ou non comme inévitables, toutes ces opinions font varier le sentiment des individus d'être plus ou moins personnellement touchés par les inégalités beaucoup plus faiblement que ne le fait par exemple le niveau de revenu du ménage..$^{15}$

Le tableau 6, qui synthétise l'ensemble des effets que nous avons examinés en prenant en compte simultanément les variables explicatives de position sociale, de «frustration relative» et d'attitudes idéologiques, fait bien apparaître deux registres explicatifs très différents de la perception des inégalités sociales : le premier s'applique aux inégalités sociales globales - pour juger de leur force et de leur degré d’acceptabilité -, l'autre s’applique aux inégalités personnellement subies (telles que les perçoivent les individus).

Le tableau montre que le registre global d’appréhension des inégalités est principalement lié à des orientations en matière de justice, tandis que le registre personnel est exclusivement associé à la position des individus dans la stratification sociale et à un moindre degré de certaines caractéristiques «assignées » de la position sociale.

I5 Le «F» de Fisher d'une analyse de variance sur le score d'impact ressenti des inégalités est de 5,I lorsque ce score varie en fonction des types de principes de justice et de 66,4 lorsqu'il varie en fonction des quintiles de revenu (pour le même nombre de degrés de liberté). 
Tableau 6. L'effet des variables de position sociale et des variables idéologiques sur la perception des inégalités sociales générales et personnelles

\begin{tabular}{|c|c|c|c|}
\hline & \multicolumn{3}{|c|}{ VARIANCE PAR DEGRÉ DE LIBERTÉ } \\
\hline & FORCE & ILLÉGITIMITÉ & IMPACT PERSONNEL \\
\hline SEXE & $6,1 * * * *$ & - & $2,2 *$ \\
\hline ÂGE & I,7* & - & $1,6 * *$ \\
\hline ORIGINE ETHNIQUE & - & $3,0 * *$ & 7,0 ***** \\
\hline ACTIVITÉ & - & $2,0 * * *$ & $5,6 * * *$ \\
\hline PCS & - & - & 4,4 ***** \\
\hline NIVEAU D'ÉDUCATION & - & $2,6 *$ & 16,9 ***** \\
\hline REVENU & - & - & 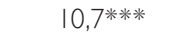 \\
\hline RÉSIDENCE & & & $7,0 * * * *$ \\
\hline FRUSTRATION RELATIVE & ।,8* & - & $6,5 * * * *$ \\
\hline ATTITUDES IDÉOLOGIQUES & 18,8***** & 10,5 ***** & - \\
\hline $\mathrm{R}^{2}$ & 0,10 & 0,06 & 0,30 \\
\hline
\end{tabular}

\section{SYNTHĖSE ET CONCLUSION}

Au terme de ces analyses plusieurs conclusions peuvent être dégagées au sujet des trois thèses que nous proposions de tester au début de cet article, celle de «l'intérêt bien compris», celle de l'effet de la frustration relative, celle de l'effet des valeurs en matière de justice sociale.

La thèse de l'intérêt bien compris n’est pas validée comme un mécanisme d'explication fort de la perception des inégalités sociales globales. Cette perception ne varie que faiblement en fonction de la position sociale occupée par les individus, même si certaines nuances apparaissent lorsqu’on distingue inégalités économiques et inégalités identitaires. En revanche, la position sociale exerce un effet très fort sur le sentiment d'être personnellement affecté par les inégalités. Le contraste est frappant: il montre que les personnes ne transposent pas leur situation personnelle à l'analyse de la société dans son ensemble. Ceux qui ont le sentiment d'être personnellement défavorisés ne conçoivent pas plus que ceux qui ont le sentiment inverse que la société dans son ensemble est fortement inégalitaire. 
La prise en compte des mécanismes de frustration relative fait bien varier de manière statistiquement significative la perception des inégalités sociales globales. Les personnes qui se comparent sont plus sensibles à ces inégalités que celles qui jugent de façon intrinsèque leur situation. Mais cet effet n'est pas très puissant et ne contribue pas à améliorer le pouvoir explicatif d'ensemble du modèle qui intègre à la fois les variables de position et la variable de comparaison sociale.

Nous avons montré par ailleurs que la perception globale des inégalités était liée beaucoup plus nettement aux principes de justice qu'à la position sociale occupée par les individus. Les ouvriers auraient pu, par exemple, être structurellement beaucoup plus sensibles aux inégalités dans la société que les cadres supérieurs. Nos résultats montrent que ce n'est pas le cas et que ce sont les orientations en matière de justice qui prennent le pas sur la «position de classe» pour faire varier cette sensibilité. Nous avons vu d'ailleurs que ces orientations en matière de justice sociale sont assez faiblement reliées à la position dans la stratification sociale. Ces conceptions de la justice exercent donc bien un effet spécifique indépendant de la stratification sociale. L’hypothèse avancée dans la première enquête du GEMAS (1976) que nous rappelions au début de cet article (selon laquelle les opinions en matière de justice sont moins déterminées par la situation objective des intéressés que par la perception qu'ils ont des inégalités existantes) nous semble donc plutôt validée.

Il resterait bien sûr à s'interroger sur la genèse de ces perceptions idéologiques, assez largement indépendantes de la stratification sociale. Nos résultats montrent qu'elles n'obéissent pas à un schéma de classes: les riches ne produisent pas l'idéologie qui devrait servir leurs intérêts. Il faudrait sans doute pouvoir tenir compte de l'histoire familiale, des effets de la transmission intergénérationnelle et d'effets de contextes locaux ancrés dans l'histoire longue; ce que l'enquête sur laquelle nous nous sommes appuyés ne permet pas. 


\section{BIBLIOGRAPHIE}

BOUDON R., 1977, Effets pervers et ordre social, Paris, PUF.

CHERKAOUI M., 1988, «Mobilité sociale et équité», Revue française de sociologie, 29-2, p. 227-245.

DELLA FAVE L. R., 1974, «On the Structure of Egalitarianism», Social Problems, 22- 2, p. $199-213$.

FORSÉ M., GALLAND O. (éds), 20II, Les Français face aux inégalités et à la justice sociale, Paris, Armand Colin.

FORSÉ M., PARODI M., 2007, «Perception des inégalités économiques et sentiment de justice sociale», Revue de l'OFCE, I02, p. 483-540.

GEMAS, 1976, Perception des inégalités sociales et de la justice sociale, Rapport pour le Commissariat général au plan.

GIJSERBTS M., 2002, «The Legitimation of Income Inequality in State-socialist and Market Societies», Acta Sociologica, 45, p. 269-285.

GODECHOT O., GURGAND M., 2000, «Quand les salariés jugent leur salaire», Économie et statistique, 331, p. 3-24.

GRUNBERG G., SCHWEISGUTH E., 1997, «Recompositions idéologiques», in L'Électeur a ses raisons, D. Boy et et N. Mayer (éds), Paris, Presses de Sciences Po, p. 139-179.

HADLER M., 2005, «Why Do People Accept Different Income Ratios? A Multilevel Comparison of Thirty Countries», Acta Sociologica, June, 48-2, p. I31-154

HAIDER F., 1958, The Psychology of Interpersonal Relations, N.Y., Wiley.

HUBER J., FORM W. H., 1973, Income and Ideology, N.Y., Free Press.

HOMANS G. C., 1974, Social Behavior. Its Elementary Forms, N.Y., Harcourt Brace Jovanovich.

JASSO G., 1978, «On the Justice of Earnings. A new specification of the justice evaluation function », American Journal of Sociology, 83, p. 1398-1419.

KELLEY J., EVANS M.D.R., 1993, «The Legitimation of Inequality: Occupational Earnings in Nine Nations », American Journal of Sociology, 99-I, July, p. 75-125. 
KLUEGEL J.R., SMITH E.R., 2009, Beliefs About Inequality. American's Views of What Is and What Ought to Be, New Brunswick and London, Aledine Transaction Publishers.

OCDE, 2008, Croissance et inégalités. Distribution des revenus et pauvreté dans les pays de l'OCDE, Paris, OCDE.

PIKETTY T., 1999, «Attitudes Toward Income Inequality in France: Do People Really Disagree?», document de travail CEPREMAP, n 9918, mai.

RAWLS J., 197I (trad. fr. 1987), Théorie de la justice, Paris, Seuil.

ROSS L., 1977, «The intuitive psychologist and his Distortions in the Attribution», Advances in Experimental Social Psychology, 10, p. 174-220.

RUNCIMAN W. G., 1966, Relative Deprivation and Social Justice, London, Routledge and Kegan Paul.

SHEPELAK N. J., 1987, «The Role of Self-Explanations and Self-Evaluations in Legitimating Inequality», American Sociological Review, 52-4, p. 495-503.

STOLTE J.F., 1983, «The Legitimation for Structural Inequality: Reformulation and Test of the Self-Evaluation Argument», American Sociological Review, 48-3, p. 33I-342.

STOUFFER S.A., SUCHMAN E.A., DE VINNEY L. C., STAR S.A., WILLIAMS R.M., 1949, The American Soldier: adjustment during army life, vol. I, Princeton, Princeton University Press. 
